\title{
Juegos del lenguaje Creación y diversidad en la organización de relatos de niños y niñas de 5 a 13 años de edad en escuelas públicas de Bogotá'
}

\section{Flor Alba Santamaria Valero ${ }^{2}$}

Docente e investigadora Universidad Distrital Francisco José de Caldas

Directora Cátedra Unesco en Desarrollo del niño

Resumen

Esta investigación trata del análisis de relatos narrados por niños de las escuelas públicas de Bogotá, Colombia, a partir de experiencias vividas. La muestra la constituyen 50 relatos analizados teniendo en cuenta aspectos referidos a las constantes y variaciones en su organización, es decir, la estructura de los relatos, así como los mecanismos que los niños utilizan para darles la heterogeneidad y șingularidad que éstos poseen. De otra parte, se identificaron también los temas abordados por los niños en los relatos y las relaciones que se establecen con el mundo cultural y social. Se demuestra igualmente en este estudio los diversos procedimientos empleados para expresar las relaciones espaciotemporales que constituyen la variedad narrativa, donde los niños aparecen en su verdadera dimensión de autores y creadores de sus relatos.

El trabajo de investigación se realizó en la localidad de Ciudad Bolívar, caracterizada, entre otras problemáticas por los altos niveles de pobreza y de violencia intrafamiliar. Aunque nuestro objetivo no fue indagar acerca del lenguaje en el medio escolar, los relatos se registraron en algunas escuelas del sector.

\section{Palabras clavé}

Narrativas, heterogeneidad, singularidad, variedad narrativa.

\section{ABSTRACT}

This investigation is dedicated to the analysis of narratives of real stories told by schoolchildren from public schools of Bogotá, Colombia. Over a corpus of 50 stories, we analyse the consistencies and differences in the organisation of the narratives. On the one hand, we observed the various forms used by children to structure and organise their narratives, as well as the mechanisms used to give them heterogeneity and singularity. On the other hand, we identified themes and their relations with the cultural and social world. We also show the various processes used by the children to express events, time, space and the spatial/temporal relationships bringing narrative variation whereby the child acquires a genuine dimension as an author and creator of his/her narratives.

\section{KEY WORDS}

Narratives, heterogeneity, singularity, narrative variation.

RECIBIDO: MARZO 2006 • ACEPTADO: ABRIL 2006

1 Síntesis de la investigación realizada por la autora con la cual obtuvo el título de doctora en Ciencias del Lenguaje en la Universidad de Paris V - Sorbona, bajo la dirección de la doctora Anne Salazar-Orvig.

${ }^{2}$ Licenciada en lingüística y literatura. Magister en Lingüística general y aplicada, Doctora en Ciencias del Lenguaje. 


\section{Introducción}

$\mathrm{E}$ 1 interés por el estudio del relato en el niño surge del trabajo realizado, de una parte, como profesora de niños en escuelas públicas de Bogotá y posteriormente como docente de la Universidad Distrital, en programas de formación de maestros, a nivel de pregrado y postgrado ${ }^{3}$. De otra parte, la participación en investigaciones sobre el lenguaje en el medio escolar ${ }^{4}$, que permitieron comprender la complejidad y variedad del lenguaje infantil y específicamente de las narrativas infantiles y su relación con los medios de información y comunicación. En este contexto, el relato se nos presenta como una forma privilegiada de acceder al mundo de los niños, ya que través de éstos podemos indagar acerca de la mirada que tienen ellos sobre sí mismos y sobre su entorno.

La población la constituyen escolares de diferentes grados de la escuela primaria, seleccionados teniendo en cuenta su deseo de narrar voluntariamente a su profesor y a un grupo de compañeros de clase, algo que le hubiese sucedido y que recordaran por haber sido agradable o desagradable. Igualmente se les solicitó expresar si el acontecimiento vivido les produjo miedo, tristeza, alegría etc.

No todos los niños narraron experiencias donde estuviesen implicados directamente; algunos contaron hechos que les ocurrieron a otros $y$ en los cuales ellos fueron testigos.

Los relatos fueron registrados durante 4 años (1998-2002). De la totalidad de 300 registros se seleccionaron cincuenta (50), (18) de niños y (32) de niñas. Se tuvieron en cuenta para la selección los relatos que fueran claros y audibles y que reunieran ciertas características en su estructura, como por ejemplo que contaran con las partes de las que habla Labov (1978) en su traba-

3 Licenciatura en Educación para la infancia, Licenciatura en Pedagogía infantil y Especialización en Infancia, Cultura y Desarrollo.

4 Como las siguientes: "La argumentación en el discurso del maestro", "La interacción maestro alumno", que abrieron el camino para la creación del grupo de investigación "Lenguaje, discurso y saberes", clasificado en categoría A en Colciencias. jo sobre la transformación de la experiencia a través de la sintaxis narrativa. A partir de la muestra se realizó solo la trascripción de lo oral a lo escrito, no se tuvieron en cuenta otras formas de expresión que se encontraron en los relatos como las tonalidades y altibajos de la voz, las pausas, los suspiros, las expresiones corporales, que son abundantes en la muestra, pero que no constituían objeto de la presente investigación.

\section{Principales elementos teóricos}

Para referirnos a los planteamientos que expondremos a continuación retomaremos los planteamientos desarrollados especialmente por el grupo de investigadores del $\mathrm{LEAPLE}^{5}$, entre otras por François (1984, 1990, 1993, 2004).

El enfoque basado en la necesidad de estudiar las conductas lingüísticas de los niños, teniendo en cuenta la diversidad discursiva en sus producciones orales, fue planteada por François y otros. (1984:7) expresan esta intención cuando dicen que: Aprendre à parler et à comprendre, c'est aprendre bien autre chose que du lexique et des structures grammaticales: en gros c'est apprendre les différents types d'enchaînement des énoncés sur le discours de l'autre ou sur mon propre discours, c'est entrer dans différents jeux de langage tant par rapport a la réalité qu'au discours de l'autre ou au discours de moi, c'est savoir alternativement répondre, raconter, argumenter, comparer.

En cuanto productor de sus propios discursos el nïño se puede considerar también como autor, este autor tiene múltiples posibilidades de organizar su producción y de crear la heterogeneidad tanto de personajes como de movimientos discursivos, François, F. (1993: 185) sostiene que al tomar al niño como autor se hace necesario mirar en qué es igual a los otros y en qué no lo es:

il participe de la généralité de la grammaire ou des savoirs partagés, insister surtout sur le mode esthétique particulier de son texte. Cette particularité n'exclut pas des rapprochements, des genres de textes communs ... la mise ensemble dans l'espace

\footnotetext{
5 Laboratoire d'Etudes sur l'Acquisition et la Pathologie du Langage chez l'Enfant: Université Paris 5.
} 
de jeu de ce qui n'est pas destiné á être ensemble entraîne le lecteur lui aussi dans un mouvement d'ouverture. Mais chaque auteur le fait á sa façon. Et il faut noter d'ailleurs que l'enfant auteur comme l'adulte auteur sé ressemble á lui-même.

Para abordar las producciones langagières de los niños con un enfoque lingüístico diferente al tradicional, François (1993) habla de "une linguistique de la circulation du discours". Circulación que puede ser material (el niño modifica el discurso del adulto); circulation "idéale" de la signification, cuando una forma lingüística utilizada en otras situaciones diferentes a las que ella ha sido recibida significa de una manera diferente; circulación monologique cuando lo que hace el sentido es la copresen-

El relato se nos presenta
como una forma privilegiada
de acceder al mundo de los
niños, ya que a través de éstos
podemos indagar acerca
de la mirada que tienen ellos
sobre sí mismos y sobre su
entorno.
cia de dos enunciados que no han sido hechos para ser colocados juntos.

Fréderic (2004:16), en su reciente producción propone una "linguistique de l'événement". Dire que les discours sont desévénement privilégie leur appréhension comme faits singuliers, plutôt que comme modeles. Esta concepción privilegia también la manera de ir de los acontecimientos a las posibles generalizaciones antes que partir de estructuras establecidas para llegar a sus componentes.

En el caso específico de los textó de los niños, François (1994:186) agrega que: Nos relations aux textes d'enfants illustre au mieux la distance émetteur-récepteur. Nous ne pouvons pas les percevoir comme lui. De même que nous nous demandons comment un enfant "digère " ce quion lui a raconté, le re-raconte, l'intègre à une rêverie, un rêve ou un cauchemar ou.. Ne pas savoir ce qu'il est vraiment serait une des caractéristiques fondamentales $d u$ texte. Pour une part un texte peut être perçu comme un monument, il arrête l'attention, se caractérise par sa clôture. Il est événement.

En esta perspectiva, François sitúa sus trabajos en una lingüistica de texto y de sus efectos de sentido.

En cuanto al relato, lo considera como discurso al concebir que los efectos de la puesta en palabras de un locutor encuentran la interpretación favorable de un receptor; por lo tanto, el discurso no puede considerarse como la simple expresión de significaciones discretas, codificadas o decodificables.

En cuanto al sentido, añade François que éste sólo se percibe a través de los movimientos discursivos que revelan tanto afinidades como rupturas. Los relatos presentan una puesta en palabras altamente heterogénea donde su diversidad es la que proporciona los elementos para el análisis.

En cuanto a la riqueza de la heterogeneidad de los relatos de los niños debe tenerse en cuenta el papel de las teorías para poder dar cuenta de lo que es particular a cada relato y de lo que tienen en común entre ellos; tales elementos se pueden mirar en su estructura y en sus formas de organización. Al respecto agrega François (2004:126): On peut aussi soupconner qu'aucune théorie ne peut vraiment se tenir «à la hauteur» de la diversité incroyable des récits et des façons de les recevoir. Non qu'il s'agisse de faire l'éloge d'un pur particulier, objet, on l'a dit, en fait fictif. Il s'agit plutôt, face au particulier, d'articuler ce qui fait qu'il est (plus ou moins) comme les autres, quion y retrouve du même et ce qui conjointement le rend spécifique. Ce qui pourrait se retrouver dans la problématique des structures et de la créativité.

Para indicar los parámetros productores de heterogeneidad, François recurre a las dicotomías; por ejemplo, lo que en el relato es universal o cultural, central o periférico, necesario o facultativo, precodificado o inventado, planificado o espontáneo, fonológico o dialógico, extraño o familiar, digno o no de ser contado.

Es porque es necesario fijar un punto de vista que ciertos hechos son seleccionados por el narrador como acontecimientos, que otros aparecen como circunstancias es así como se construye una estructura narrativa. Se puede decir que son raros los hechos notables por sí mismos; es la forma de enfocarlos y de contarlos lo que les confiere un valor narrativo.

Análizar los discursos infantiles globalmente es hacerse las mismas preguntas que para el discurso de los adultos. No se trata de definir 
una manera infantil de contar, sino de hacerse ciertas preguntas.

Una síntesis de algunos aspectos importantes a tener en cuenta en el estudio del relato infantil en las que coinciden François y su grupo son:

- Los niños no cuentan como lo hacen los adultos $\mathrm{y}$, por lo tanto, construyen relatos reconocidos como tales por sus interlocutores, no importa quiénes sean

- Es lo particular lo que constituye el interés de intercambio y no el hecho de decir la misma cosa.

- Las formas efectivas de contar reenvían a los subgéneros, como mezclas de secuencias discursivas, más que a los géneros puros.

- Contar aparece como una práctica más compleja y más diversa cuando no se hace de la manera como lo hacen los adultos, sino a partir de datos de las producciones infantiles.

- Tener en cuenta las diferencias en el relato niño/niño, como también entre niño $\mathrm{y}$ adulto.

A partir de los planteamientos teóricos expuestos anteriormente surgen cuestionamientos a los que se pretende dar respuesta a partir del análisis del corpus seleccionado.

\section{Perspectiva de análisis}

Para el análisis de la muestra de relatos partimos de las siguientes preguntas:

- ¿Cómo organizan y estructuran los niños sus relatos?

- ¿Cómo expresan los niños los acontecimientos, el tiempo y el espacio?

- ¿De qué hablan los niños en sus relatos?

En cuanto a la primera pregunta, se observa que los niños se presentan como narradores acudiendo a mecanismos que les proporciona la lengua para darle organización narrativa a sus historias. Algunos para situarse como narradores lo anunciaron en la apertura del relato, colocándose en situación de interacción frente a sus interlocutores, mediante expresiones de discurso tales como: "les voy a contar", "mire que un día", "les quiero contar". En algunos casos los niños hicieron un resumen de lo que iban a narrar.

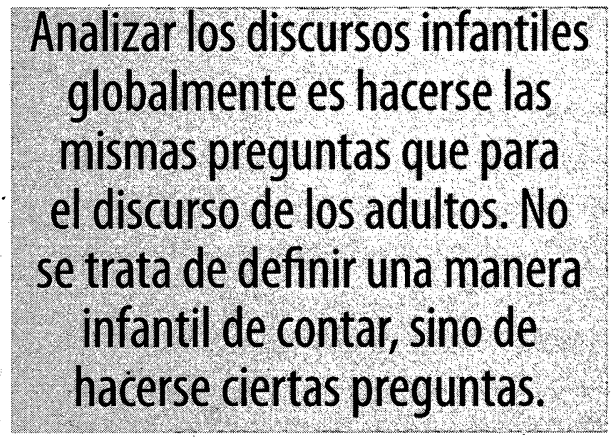

Los niños emplean también diversas formas - de concluir sus relatos; algunos cerraron sus historias con elementos que no pertenecían a la historia, pero que indicaban que ésta no continuaría (eso fue todo, no es más. Ya y listo). Otros cerraron sú historias con enunciados que no están relacionados con la secuencia narrativa pero que tienen como función suavizar un tanto los acontecimientos dramáticos como el final que da Luis Alejandro a su relato: $Y$ me eché una ollada de agua para el jugo y me quemé todo esto (señala el abdomen) y me llevaron al doctor y me echaron una pomada, y eso me quedó unas burbujas y eso me las totiaron y me quedó costra y por la noche mi papá me trajo pan y yogur.

La variedad de formas de apertura y de cierre empleadas por los niños en la organización de sus relatos, nos muestran la diversidad de mecanismos narrativos que dan a los relatos la heterogeneidad tanto en su organización como en su estructura y ponen de manifiesto que más que buscar un modelo preestablecido de organización del relato, se requiere buscar juegos y movimientos discursivos que emplean los niños como autores de sus relatos.

Los niños utilizaron también diversas formas para entrar a la secuencia narrativa y construir la intriga, estableciendo por ejemplo cierta clase de relaciones que les permitieron presentarse como protagonistas y narradores al mismo tiempo. Para ello emplearon especialmente los pronombres personales (Yo, me). Los relatos del yo fueron mayoritarios; en éstos el yo representa al narrador para hablar de sí mismo, evocando una experiencia vivida. 
Se constata en la muestra de relatos una diversidad tanto a nivel de estructura como de organización discursiva. Se puede hablar entonces de la heterogeneidad discursiva presente en los relatos y de las múltiples posibilidades de creación y de organización que expresaron los niños como autores de sus propios textos. Esta heterogeneidad está dada por el juego de posibilidades que utiliza el niño en la construcción de los relatos entre lo que es constante y lo que es diverso y. que en términos de François ${ }^{6}$, es lo que permite los efectos de interés y de placer que producen en el lector los relatos construidos por los niños.

En relación con el interrogante de cómo expresan los niños los acontecimientos, el tiempo y el espacio, se observa en la muestra que se emplean ciertas clases de verbos que están relacionados, bien sea con las acciones donde hay presencia de un agente que las realiza o con los procesos donde algo le sucede a alguien. Los eventos tienen como marco de referencia la coțidianidad.

Podemos decir que en cuanto a la ubicación del tiempo y del espacio en los relatos, constatamos sobre todo la presencia de un saber compartido con sus interlocutores y por lo tanto los niños no veían la necesidad de expresarlos, o si los expresaron, lo hicieron de manera indefinida (una vez, un día) o sin fijar un punto de referencia espacial definido (allá). Es gracias a los diferentes movimientos y juegos del lenguaje que el niño realiza con la diversidad de tiempos, de espacios y de acontecimientos que él logra darle a sus producciones, un estilo, una atmósfera, un aire particular.

En cuanto al tercer interrogante, los principales dominios temáticos en relación con los acontecimientos que narraron los niños fueron sobre todo los acontecimientos generados por seres humanos, que ejercían el rol de agentes para ejecutar determinadas acciones sobre otros seres humanos (pacientes) como es el caso de los castigos físicos y actos violentos. En efecto, en los relatos cuyo tema fue el castigo físico (pegarles) los agentes estaban representados por los padres y los pacientes por los niños.

Los niños se representaron como los $p a$ cientes, en quienes recae el castigo, sin enjuiciamiento de los hechos, lo que manifiesta que los niños aceptaron en forma pasiva el castigo.

El tema del castigo físico es reiterativo en los relatos de los niños aspecto que evidencia la frecuencia de esta modalidad de castigo, empleada en algunos sectores de la población colombiana, especialmente en los sectores populares.

El tema de la violencia parece expresarse en los relatos; de un lado, porque hace parte de la realidad social, y algunos la viven y son afectados más de cerca que otros, y de otro lado, porque los medios de comunicación se encargan de hacer énfasis diariamente en los hechos trágicos sobrecargándolos de dramatismo.

\section{EJEMPLOS}

Los niños hablan también de acontecimientos que les suceden a los seres humanos donde no hay presencia de agentes, sino solamente de pacientes a quienes les ocurren cosas. Por ejemplo, los accidentes o las muertes por causas naturales.

Sin embargo, la temática que predomina en nuestra muestra es la de los accidentes de diversa índole, lo cual parece poner de manifiesto un cierto grado de inseguridad en los distintos espacios en los que los niños interactúan.

Podemos resumir los principales dominios temáticos que predominan en los relatos de la siguiente manera.

\begin{tabular}{|l|c|}
\hline Temas & Cantidad de relatos \\
\hline Castigo físico & 10 \\
\hline Accidentes automovilísticos & 3 \\
\hline Accidentes domésticos y otros & 18 \\
\hline Muertes por causas naturales y ataques & 5 \\
\hline Actos violentos & 6 \\
\hline Acontecimientos cotidianos & 8 \\
\hline
\end{tabular}

${ }^{6}$ ENFANTS ET RÉCITS: Septentrión. France, 2004. 


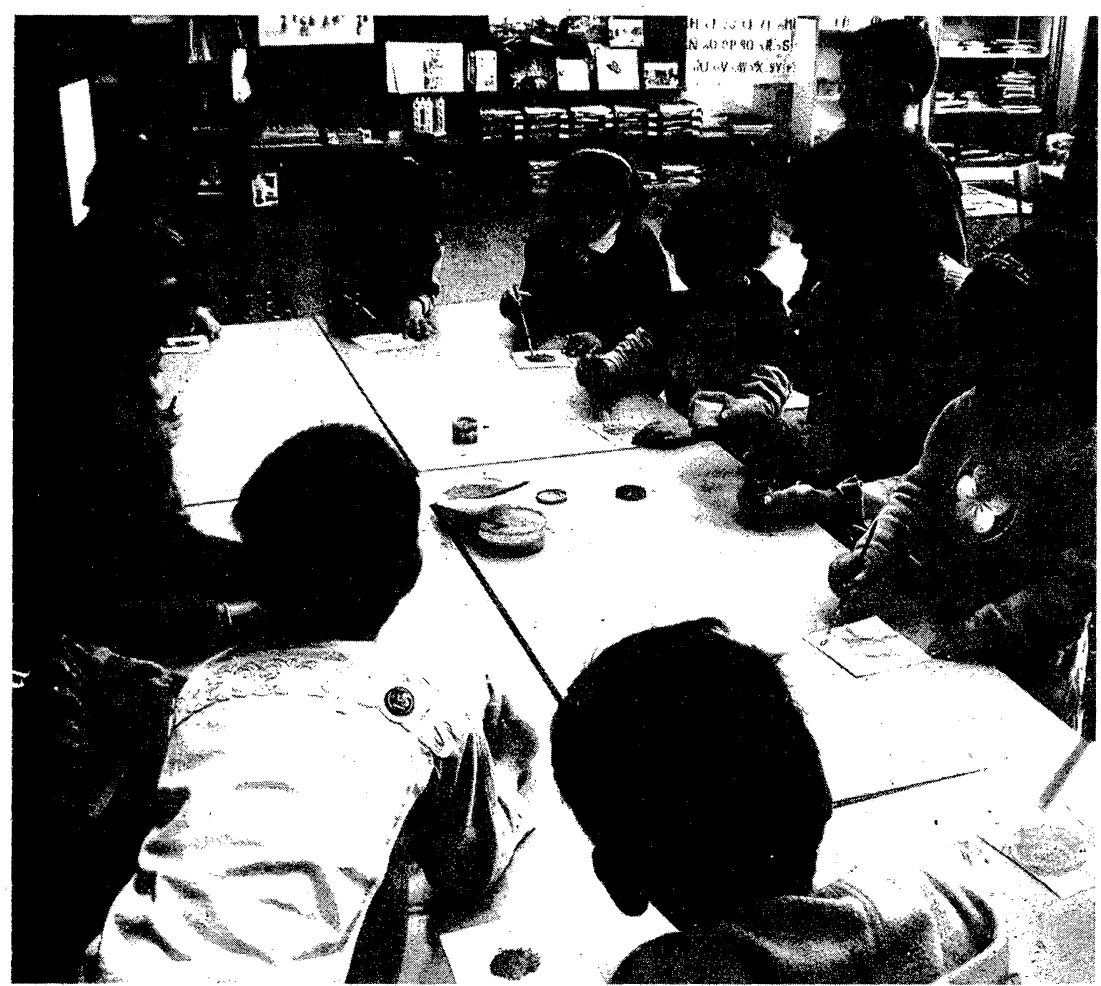

En términos generales, en la muestra de relatos encontramos diversidad de dominios temáticos y diferentes maneras de asegurar la continuidad textual. Así por ejemplo, hay niños que en la secuencia narrativa mezclaron diferentes modos de discurso (como la descripción, la explicación, el diálogo) y otros, por el contrario, mantuvieron la secuencia narrativa sin mezclar otras formas de discurso.

A continuación presentamos un relato donde se observa una mezcla de temas (en la primera parte, la niña no quiso rezar, en la segunda, la toma guerrillera) y se puede apreciar también la mezcla de explicaciones y descripciones en la narración.

40 / cuando yo estaba en el Caquetá pues con mi abuelito y mi abuelita ese dia mis abuelitos me dijeron que rezara y yo no quería y entonces mi abuelita me dijo que no rezara entonces me acosté después por la noche empécé a oír unos disparos era la guerrilla era la guerrilla y abi si mi abuelito y todos nos asustamos y ahi si yo quise rezar empezamos a rezar y mi abuelito se fue a ver la puerta de atrás porque nosotros teniamos una cochera, teniamos marranos después empezaron los disparos después al otro día eb ya amaneció todo estaba calmado y yo llegué en pijama me sali por la ventana una ventanita chiquita y fui a ver los muertos bastante gente habian matado a un policía y muchos señores y muchos policías de todo después yo sali yo llegué me dijo usted qué hace aqui yo le dije quiero mirar acá los muertos dijo váyase para la casa yo no le hice caso y me quede mirando después llegué yo y me fui para la casa y ya. Leidy, 9 años. Tercero de primaria.

\section{Conclusión}

La investigación permitió comprender la actividad narrativa de los niños y especialmente la especificidad de la organización de los relatos en donde se mezclan diversos movimientos discursivos y juegos del lenguaje para construir el escenario de la significación. Desde este punto de vista, son evidentes los efectos de interés y de placer, generados no solo en lo que se cuenta sino en cómo se cuenta. Es así como se encuentran relatos que pueden inspirar sentimientos de dolor, de compasión o de indignación (por ejemplo, relatos donde los padres o familiares golpean a los niños y en los que hay muertes violentas). Pero igualmente son capaces de producir risa (cuando cuentan y hacen sentir el hecho como chistoso) y de articular los dos dominios de significación en un mismo relato (cuando el hecho que se narra es trágico pero su final es inesperado y tiene como efecto el de suavizar los hechos al narrarlos).

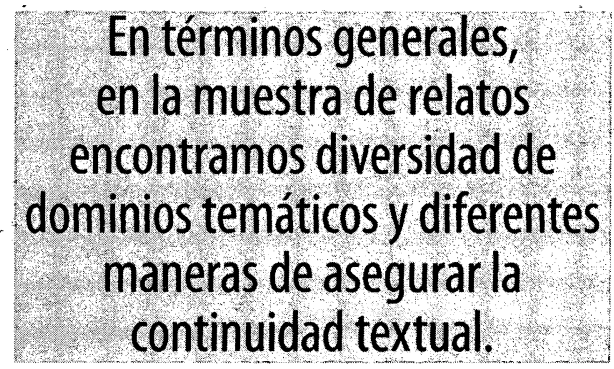

Lo anterior manifiesta la diversidad de estrategias utilizadas y la manera como el niño se sitúa ante los hechos en el relato.

En cuanto a la estructura, en la muestra de relatos priman los relatos cortos, sin muchas localizaciones espaciales, ni tampoco temporales, y donde el relato, fundamentalmente, se organiza a partir de una secuencia de acciones. Lo anterior significa que como muchos de los eventos a los que se refieren los niños son dramáticos 
por sí mismos, por lo tanto no necesitan complejizarlos con evaluaciones o con modalizaciones. En consecuencia, se puede decir que se ponen en ejecución estructuras de relatos para ser èscuchados para ser visualizados más que para ser leídos, que se semejan un tanto a guiones de cortometrajes para películas de acción.

En los acontecimientos narrados los niños se representan el mundo en relación cercana con los adultos, que pueden ser familiares o no familiares. En estas relaciones son los adultos quienes tienen siempre ingerencia sobre ellos y en consecuencia, los niños no se dibujan en sus relatos como seres autónomos ni mucho menos como dueños de sus propios actos. Por el contrario, se representan como pacientes que sufren una realidad y se expresan de manera simple y directa acerca de las acciones de los adultos.

La indefinición del tiempo y del espacio, presentes en buena parte de los relatos, dejan abierta la posibilidad de que los hechos vividos que cuentan los niños sean situaciones generalizadas, que refuerzan un tanto la imagen de niño como ser pasivo que vive eventos difíciles que corresponden más bien al mundo de los adultos pero que a él se les imponen por obligación.

El relato tiene una importancia grande para que el niño pueda interpretar su propia vida, al hablar de lo que le sucede. De esta manera, el niño es capaz de expresar no solo su mundo interno sino también poner en evidencia las relaciones que establece con los otros o que los otros establecen con él. Es importante reconocer que, culturalmente, en Colombia se tiene una experiencia narrativa muy rica y en general se otorga sentido a la vida, a través del relato. En este sentido, el relato acompaña la vida de los niños, como

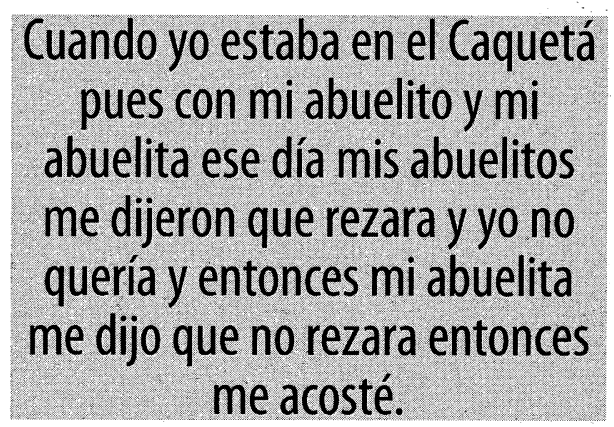

práctica abierta y espontánea de comunicación, en donde predomina el chiste y la cotidianeidad.

Esta visión carnavalesca de la vida contribuye de alguna manera a que los niños se conozcan y se encuentren con el otro compartiendo el placer de contar y de ser escuchados. En efecto, en el contexto social y cultural en donde se sitúa la investigación, es por medio de la palabra que los niños expresan la dureza de su condición de vida, la violencia permanente, la adversidad, entre otras realidades y más claramente la coerción y el poder que los adultos ejercen sobre ellos.

Como una proyección de la presente investigación se plantea, por parte del Grupo de investigación Lenguaje, Discurso y Saberes, realizar un estudio comparativo de la imagen que los niños tienen de sí mismos y la imagen que los adultos tienen de los niños, en diferentes niveles sociales del contexto colombiano. Ó

\section{Bibliografía}

ADAM, J.M. 1983. Le texte narratif, Nathan Université. Paris. 1984: Le récit, Que Sais-je? No 2149, P.U.F., Paris. 2001. Les textes: types et prototypes. Nathan. Paris.

ADAM, J.M. y LORDA, Clara, 1999 B. Lingüistica de los textos narrativos. Ariel Lingüística. Barcelona.

ANDRADE G., 1996. "L'insertion sociale par l'acquisition de la langue». In: Le Plurilinguisme Spontané Des Habitants de Belleville et de la Goutte D'or. Septentrion. 132193. Villeneuve d'Ascq Cédex.

ARIES, P., 1993. El niño y la calle, de la ciudad a la anticiudad. 283-310. En: Ensayos de la memoria, 1943-1983. Norma. Bogotá.

AUTHIER-REVUZ, J. 1998. «Enonciation, méta-énonciation Hétérogénéités énonciatives et problématiques du sujet ». 63-79. In : Les sujets et leurs discours. Publications de L'université de Provence. Aix-en Provence.

BACHELARD, G. 1965. La poética del espacio. Fondo de Cultura Económica. México.

BAJTIN, M. 1977. Le marxisme et la philosophie du langage. Minuit. 
1978. Esthétique et théorie du roman, Gallimard, Paris.

1982. El problema de los géneros discursivos. En: Estética de la creación verbal, Siglo XXI. 48-293. México.

BALLY, CH. 1977. El lenguaje y la vida. Editorial Losada. S.A. Séptima edición. Buenos Aires.

BARDIN L. 2001. Lanalyse de contenu. PUF, Paris.

BAYLON CH, MIGNOT X. 1995. Sémantique du Langage. Nathan, Paris.

BARTHES, R. 1985. L'aventure sémiologique, Seuil. Paris.

BERTAUX D. 1997. Les récits de vie. Nathan Université. Paris.

BENVENISTE, É. 1974. Problèmes de Linguistique Générale 2. Gallimard.

1966. De la subjectivité dans

le langage. In: Problèmes de linguistique générale. Gallimard, 258-266.

BOIRON V. 2001. Deux moments d'un dialogue narratif dans une classe maternelle. Affinités thématiques et mouvements discursifs: continuités et déplacements, diversité des places discursives. CALAP, n ${ }^{\circ}$ 21-22, pp. 17-39.

BOOTH W. 1977. Distance et point de vue. In: Poétique du récit, editions du Seuil. Paris. 85113.

BRES, J. 1994. La narrativité. Duculot S. A. Gembloux (Belgique).

1993. (sous la direction) Le récit oral. Université Paul Valery - Montpellier III.

BOSQUE, I y DELMONTE, V. 1999. 'Gramática descriptiva de la lengua española. Real Academia Española, Espasa Calpe, S.A. Madrid.

BRONCKART, JP. 1983. Psycholinguistique de l'enfant. Delachaux et Niestlé. Paris. 1993. Lorganisation temporelle des discours. Langue française No 97. pp. 3-13.

1997. Activité langagière, textes et discours, Lausanne, Delachaux \& Niestlé. Paris.

BRUNER, J. 1997. Là educación; puerta de la cultura. Visor S.A. Madrid.

1991. Actos de significado. Alianza Editorial S.A. Madrid.
1996. The Piaget-Vygotsky

$\&$ the Growing Mind. Memorias, Viena. 2002. Pourquoi nous racontons nous des histories? Retz éditions. Paris.

CABREJO, E. 2004. "La cadena simbólica de la lengua”. En: revista Magisterio. Bogotá, pp. 5-11.

1992. «L'enfant et la deixis», La deixis, MOREL M.A., DANONBOILEAU L. (Eds), Paris, P.U.F., pp. 409414.

CALSAMIGLIA H, TUSON A. 1999. Las cosas del decir. Ariel. Barcelona.

CASAS, F. 1998. Infancia: Perspectivas psicosociales. Paidós. Barcelona.

CUERVO, R. 1955. Apuntaciones criticas sobre el lenguaje bogotano. $9^{\circ} \mathrm{ed}$. Instituto Caro y. Cuervo. Bogotá.

CHAFE, W. y NICHOLS, J. 1986. Evidentially: The Linguistic Coding of Epistemology, vol. XX, Norwood, N. J, Ablex.

CHAFE, W. 1970. Meaning and the structure of language. Chicago.

1976. Significado y estructura de la lengua. Editorial Planeta. Barcelona.

CHARAUDEAU P, 1983. Langage et Discours. Éléments de Sémiolinguistique. Hachette, Paris. . 1992. Gramaire du sens et de l'expresion. Hachete, Paris. 1993. L'acte narratif dans

les interlocutions. In: Le récit oral. Praxilin Université Paul Valery. 23-35. Montpellier III.

DELAMOTTE-LEGRAND, R. 1997. «Reprises, impositions, résistances et autres interventions. Enfants de 4 ans et adultes face au récit», CALAP n ${ }^{\circ} 14$, pp. 59-77.

2001. «L'activité narrative du jeune enfant", Le journal des Professionnels de l'Enfance, n' 9, pp. 52-53.

2004. Ce que les récits enfantines nous apprennent sur le récits et reste. In: Enfants et Récits Mises en mots et "reste":13-40, Presses Universitaires du Septentrion, France.

DUCROT, O. 1972. Dire et ne pas dire. Principes de sémantique linguistique, Paris, Hermann 1984. Le dire et le dit. $\mathrm{Mi}^{-}$ nuit. Paris. 
1990. Polifonia y argumen-

tación. Universidad del Valle, Cali.

EGLE, B. DOMINIQUE, J. 1998. Histoire de l'enfance en occident. Tome I. Éditions du Seuil. Bari.

FAYOL, M. 1985. Le récit et sa construction, une approche de psychologie cognitive, Delachaux et Niestlé, Paris, Seuil.

FRANÇOIS F., FRANÇOIS D., SABEAUJOUANNET, SOURDOT M. 1977. La Syntaxe de l'enfant avant 5 ans. Larousse, Paris.

FRANCOIS, F. 1980. "Linguistique et analyse du texte”. In: Linguistique, PUF, 233-277. Paris.

.1993. «L'enfant et les récits». En: pratiques de l'oral. Nathan-Pédagogie.149223. Paris.

1998. Le discours et ses entours. L'Harmattan. Paris. HUDELOT C, ET E. SABEAU - JOUANNET. 1984 Conduites linguistiques chez le jeune enfant, PUF. Paris.

1990. La communication inégale. Heurs et Malheurs de l'interaction Verbale. Delachaux et Niestlé. Paris.

1996. Origène du langage. En: Origines, identités, destinées. Que dire à un enfant qui s'inquiète de son origine. ESF éditeur. Paris.

1988. Le récit et ses normes. 217241. In: La langue française est elle gouvernable? normes et activités langagières. Delachaux et Niestlé. Neuchâtel, Paris. . 2004. Enfants et Récits, Mises en mots et "reste". Textes choisis et présentés par DELAMOTTE-LEGRAND. Presses Universitaires du Septentrion, France.

FILLMORE, C.J. 1968. The case for case, Universals in Linguistic Theory. Bach y Harms, New York: Holt, Rinehart and Winston.

FOUCAULT, M. 1990. La arqueología del saber. Siglo XXI. México.

GALINDO, J. 1997. Sabor a ti. Metodología cualitativa en investigación social. Universidad Veracruzana. México.
1998. Técnicas en Investiga-

ción en sociedad, cultura y comunicación. Addison Wesley Longman. México.

GARCÍA MÁRQUEZ, G. 2003. La patria amada aunque distante, mensaje con motivo de los 200 años de la Universidad de Antioquia. Medellín.

1995. Un manual para ser niño. Ministerio de Educación Nacional. Bogotá.

1995. Cómo se cuen-

ta un cuento. Voluntad. Santa Fe de Bogotá.

GARCÍA, M. Tordesillas C. 2001. La enunciación en la lengua. De la deixis a la polifonía. Gredos. Madrid.

GOFFMAN, E. 1997. La presentación de la persona en la vida cotidiana. Amorrortu Editores. Buenos Aires.

GOSSELIN, L. 1996. Sémantique de la temporalité en français. Duculot S.A. Louvainla-Neuve.

HICKMANN, M. 2000. Le développement de l'organisation discursive, in Lacquisition du Langage, Presses Universitaires de France, pp. 83-115.

HUDELOT, C. 1987. «Organisateurs discursifs du dialogue adulte-enfant: une esquisse tracée à partir de quelques dialogues (enfants de 5 à 6 ans)», Modèles linguistiques, IX, 1, pp 33-51

HALLIDAY, M. A. 1982. El lenguaje como semiótica social. Fondo de Cultura Económica. México.

. M.A.K., Hasan. 1976. Cohesion in English, London, Longman.

HELER, A. 1985. Historia y vida cotidiana. Grijalbo. México.

KERBRAT- ORECCHIONI C. 1990. Les interactions verbales. Tome I, Armand Colin, Paris.

1980. L'Énonciation. De la subjectivité dans le langage. Arman Colin. Paris.

KINTSCH, W. et VAN DIJK, T.A. 1978. Towards a model of discourse comprehension and production, Psychological Review, 85, 363-349. 
VAN DIJK T.A. 1975. Comment on se rappelle et on résume des histoires, langages 40, 98-116.

JUNG, C. 1999. Recuerdos, sueños, pensamientos. Seix Barral. Barcelona.

LAFOREST, M. 1996. (Sous la direction) Autour de la narration. Centre International de Recherche en aménagement linguistique (CIRAL). Université Laval. Nuit Blanche. Québec.

LABOV, W. et Waletsky, J. 1967. Narrative analysis: Oral versions of personal experience, dans June Helm (dir.). Essays on the verbal and visual arts, American Ethnological Society, University of Visual Arts, American Ethnological Society, Seattle, University of Washington. Press, 12-44.

1988. La transformación de la experiencia en sintaxis narrativa. Centro de traducciones Universidad del Valle. Cali.

LYONS, J. 1980. Semántica. Editorial Teide, Barcelona.

MAINGUENEAU, D. 1998. Analyser les textes de communication, Dunod, Paris.

MERLEAU-PONTY,M.1997. Fenomenología de la percepción. Ediciones Península. Barcelona.

MONDADA, L. 1994. Verbalisation de l'espace et fabrication du savoir: Approche linguistique de la construction des objets de discours. Université de Lausanne, 2000. Linguistique et paroles urbaines. En: Décrire la Ville. 59-84. Paris.

MOESCHLER, J. 1996. «Récit, ordre temporal et temps verbaux». En: Autour de la narration. Nuit Blanche. 151- 171. Québec.

OLSON, D. Torrance, N. 1995. Cultura escrita y oralidad. Editorial Gedisa. España.

ONG, W. 1994. Oralidad y escritura. Tecnologias de la palabra. Fondo de Cultura Económica. Bogotá.

PELÁEZ, G. "El niño y la violencia: Una perspectiva actual". En: Infancias imágenes No 2. Noviembre de 2002. Universidad Distrital Francisco José de Caldas. Bogotá.

PHILIPPE, J. 1996. LAAutobiographie. Nathan Université. Paris.

RECANATI, F. 1998. «Le paradoxe de la première personne». pp. 7-17. En: les sujets et leurs discours. Enonciation et interaction.
Publications de I' université de Provence. Aix-en Provence.

REY, A. SANTAMARÍA, F. GORDILLO, A. TAPIERO, O. 2000. La argumentación en el discurso del maestro. IDEP. Universidad Distrital Francisco José de Caldas. Bogotá. RICCEUR, P.1980. La narrativité, Editions du CNRS, Paris. Seuil. Paris.

1983. Temps et récit, vol 3, 1990. Soi - même comme un autre. Éditions du seuil. Paris.

ROGOFF, B. 1993. Aprendices del pensamiento. Paidós. España.

ROULET, E. 2001. Un modèle et un instrument d'analyse de l'organisation du discours, Peter Lang.

SALAZAR ORVIG, A. 1999. Les mouvements du discours. Style, référence et dialogue dans des entretiens cliniques. L'Harmattan. Paris.

2000. La reprise aux sources de la construction discursive, Langages, $\mathrm{N}^{\circ}$ 140 , pp. 68-91.

2002. Remarques sur la continuité et l'engagement dialogique chez le jeune enfant in DANON-BOILEAU L., HUDELOT C., SALAZAR ORVIG A., (Eds) Usages du langage chez l'enfant, Paris, Ophrys, Bibliothèque des Faits de langue, pp. 61-81.

. et Hudelot, C. 1989. Enchaînements, continuités et déplacements dialogiques chez le Jeune enfant. XII, 1, 99-115, Verbum.

SANTAMARÍA, F. 2002. "Niñez: Su circunstancia colombiana y la educación en el arte de convivir". En: Revista Infancias Imágenes No 2. Universidad Distrital Francisco José de Caldas. Bogotá. 2002. "Narratividad infantil: E1 relato oral". Encuentro de Investigación. Universidad Distrital Francisco José de Caldas. IEIE. Bogotá.

SAUSSURE, L. 2003. Temps et pertinence. De boeck. Duculot. Bruxelles.

SCHUTZ, A., Le chercheur et le quotidien. phénoménologie des sciences sociales, Paris, Méridiens Klincksieck, 1987, 286 p. Trad. fr. par. 
A. 1974. El problema de la realidad social. Amorrortu Editores. Buenos Aires.

1993. La construcción significativa del mundo social. Ediciones Paidos/ Recherche. Barcelona.

SIMONE; R. 1992. Diario lingüístico de una niña. Gedisa. España.

VAN DIJK, T. A. (compilador). 2000. El discurso como interacción social. Gedisa.

1980. Estructura y funciones del discurso. Siglo XXI Editores, México.

VERÓN, E. 1987. La semiosis social. Gedisa. Buenos Aires.

VET, CO. 1980. Temps, aspect, et adverbes de temps en Français contemporain. Droz. Genève.

VINCENT, D. 1997. Le discours rapportéau quotidien. Nuit Blanche. Québec.
VION, R. 1992. La communication verbale. Analyse des Interactions. Paris Hachette.

1998. Du sujet en linguistique.

En: Les sujets et leurs discours, Université de Provence. 189-202. Aix-en-Provence.

VYGOTSKI, L. 1987. Pensamiento y lenguaje. Teoría del desarrollo cultural. Pleyade. Buenos Aires.

1990. La imaginación y el arte en la infancia. Ediciones AKAL, Madrid.

VUILLAUME, M: 1990. Grammaire temporelle des récits, Minuit. Paris.

WECK DE G. 1991. La cohésion dans les textes d'enfants. Delachaux et Niestlé. Paris.

WEINRICH, H. 1973. Le temps, Paris Seuil.

WINNICOTT, D. 1998. Acerca de los niños. Paidos: Buenos Aires. 